\title{
TOUTATIS: A radio frequency quadrupole code
}

\author{
Romuald Duperrier \\ CEA-Saclay, 91191 Gif sur Yvette Cedex, France \\ (Received 16 October 2000; published 7 December 2000)
}

\begin{abstract}
A cw high power linear accelerator can only work with very low particle losses and structure activation. At low energy, the radio frequency quadrupole (RFQ) is an accelerator element that is very sensitive to losses. To design this structure, a good understanding of the beam dynamics is required. Generally, the reference code PARMTEQM is enough to design the accelerator. TOUTATIS has been written with the goals of cross-checking results and obtaining more reliable dynamics. This paper relates the different numerical methods used in the code. It is time based, using multigrids methods and adaptive mesh for a fine description of the forces without being time consuming. The field is calculated through a Poisson solver and the vanes are fully described, allowing it to properly simulate the coupling gaps and the RFQs extremities. Theoretical and experimental tests are also described and show a good agreement between simulations and reference cases.
\end{abstract}

PACS numbers: 29.27.Bd, 29.27.Eg, 29.27.Fh, 41.75.-i

\section{INTRODUCTION}

A cw high power linear accelerator can only work with very low structure activation. At low energy, the radio frequency quadrupole (RFQ) is an element that is very sensitive to losses. To design the RFQ, a very good estimation of loss location and deposited energy is necessary. Until now, the reference code PARMTEQM was enough to design the accelerator. But recent studies have shown that this code could be insufficient to accurately estimate the transmission and emittance growth at the output of the accelerator [1]. A new code, TOUTATIS, has been written to describe as well as possible the beam dynamics by a numerical approach. The main advantage of this approach is the possibility of computing the electrical fields for any vanes geometry. In particular, this approach is the only way to simulate structure discontinuities as coupling gaps.

\section{TOUTATIS ALGORITHM}

The scheme used by TOUTATIS to simulate the beam dynamics in RFQ is simple. The simulation is performed using time as an independent parameter. This is the only way to calculate accurately the self-forces of a bunch of particles. For each time step, a fraction of the radio frequency period, the charge distribution $\rho$ is discretized in a 3D mesh with a "cloud-in-cell" scheme. In the same grid, the vane geometry is embedded and likened to a Dirichlet boundary. The Poisson equation is solved with the obtained grid. The solver is detailed in the following sections. Finally, forces are extracted from the potential. This scheme allows one to take into account external fields, space charge, and image effects. Forces are applied to macroparticles via the following step-to-step scheme:

Electronic address: rduperrier@cea.fr

$$
\begin{aligned}
\vec{r}_{n+1} & =\vec{r}_{n}+\vec{\beta}_{n} c \delta t+\frac{\delta t^{2}}{2} \vec{a}_{n}, \\
(\gamma \vec{a})_{n+1} & =\frac{q}{m} \vec{E}\left(\vec{r}_{n+1}\right), \\
(\gamma \vec{\beta})_{n+1} & =(\gamma \vec{\beta})_{n}+\frac{\delta t}{2 c}\left[(\gamma \vec{a})_{n+1}+(\gamma \vec{a})_{n}\right],
\end{aligned}
$$

where $\delta t$ is the time step, $\vec{E}$ is the electrical field, $r, \beta c, \gamma$, $q$, and $m$, respectively, are the position, speed, relativistic factor, charge, and mass of the particle. The vectors $\vec{\beta}$ and $\vec{a}$ are computed using the following equation:

$$
\vec{x}_{n}=\frac{(\gamma \vec{x})_{n}}{\gamma_{n}}
$$

with

$$
\gamma_{n}=\left[1+\left\|(\gamma \vec{\beta})_{n}\right\|^{2}\right]^{1 / 2} .
$$

The main advantage of this scheme is that its Jacobian is strictly equal to 1 . The code is then preserved from phoney damping of emittance which may occur with the "leapfrog" scheme [1]. This algorithm can be looped to reach any longitudinal position in the RFQ.

\section{FINITE DIFFERENCE METHOD}

In TOUTATIS, the Poisson equation is solved using the finite difference method. The purpose of this section is not to describe in detail this well-known method. The reader will find in literature many specialized books on this topic $[2,3]$. Only the main principles are presented.

In the mesh (Fig. 1), a particular node, labeled 0 , is bound to its neighbors, labeled from 1 to 6 , by a finite equation. This equation is a function of the electrical potential on each node $\Psi_{i}$, the charge density on the considered node $\rho_{0}$, and some weighed coefficients $\alpha_{i}$,

$$
\Psi_{0}=f\left(\rho_{0}, \sum_{i=1}^{6} \alpha_{i} \Psi_{i}\right)
$$




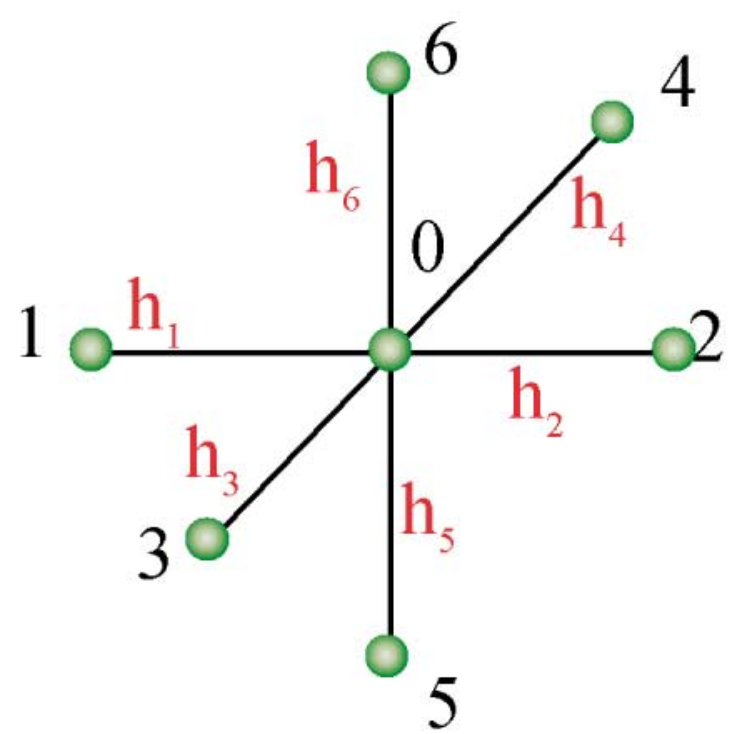

FIG. 1. (Color) Illustration of the finite difference method.

The coefficients are functions of the distance between nodes $h_{i}$.

This kind of weighting allows one to take into account the vane shape very accurately. The famous "stairs" discretization is then avoided. The principle is to compute each node of the grid with its associated equation taking into account the new values calculated for the previous nodes. Once all nodes of the mesh are computed, the scheme can be looped to reach convergence, in other words, until the values of the electrical potential do not change anymore. This particular way to use the finite difference equation is called Gauss-Seidel relaxation. The accuracy of this method is a function of only $h$. When $h$ tends towards zero, the solution becomes exact [2]. However, the convergence is slow enough to become prohibitive for the simulation of a whole RFQ with reasonable values of $h$ and $\delta t$. For instance, 1 week of computation on a Pentium $450 \mathrm{MHz}$ is necessary for the injector of proton of high intensity (IPHI) design [4]. Several methods have been developed to get acceleration of the relaxation pro- cess. We can quote the Chebyshev acceleration [5] and the Frankel-Young acceleration [2]. The next section describes the method used by TOUTATIS to reduce this computation time from 1 week to $5 \mathrm{~h}$.

\section{MULTIGRIDS METHODS}

Practical multigrid methods were first introduced in the 1970s by Brandt [6]. Basically, we need to solve the following equation:

$$
\Delta \Psi=\rho,
$$

where $\rho$ is the source term, $\Psi$ is the researched scalar potential, and $\Delta$ is the Laplacian operator. The source term is discretized in a fine grid. Performing $i$ Gauss-Seidel cycles on this fine grid, we obtain a rough estimation, $\Psi^{i}$, of $\Psi$. The Laplacian of $\Psi^{i}$ is not equal to $\rho$, the difference,

$$
\tilde{\rho}^{i}=\Delta \Psi^{i}-\rho,
$$

is called the residual or defect. This residual is the solution of a second Poisson equation dealing with the error

$$
\Delta \tilde{\Psi}^{i}=\tilde{\rho}^{i},
$$

where $\tilde{\Psi}^{i}$ is the scalar correction which allows one to get $\Psi$ via the relationship

$$
\Psi=\Psi^{i}-\tilde{\Psi}^{i} .
$$

This is an important point in multigrid methods; we are going to estimate the error after a few relaxations rather than the final solution $\Psi$ step by step. In order to get rapid estimation of this error, Eq. (7) is solved performing a relaxation process using a coarser grid, the residual having been previously discretized in this new mesh (restriction). This coarser grid is also marred by mistakes which can be estimated employing the same technique, and so on. To correct one fine grid with the result of the coarser one, an interpolation process, named prolongation, is performed. This is the main principle of the multigrid methods. The user has to combine the different stages in respect to his/her problem. This gives many possibilities of cycle architectures. We can quote the V cycle which is very common [7]. The cycle used by TOUTATIS is described in Fig. 2.

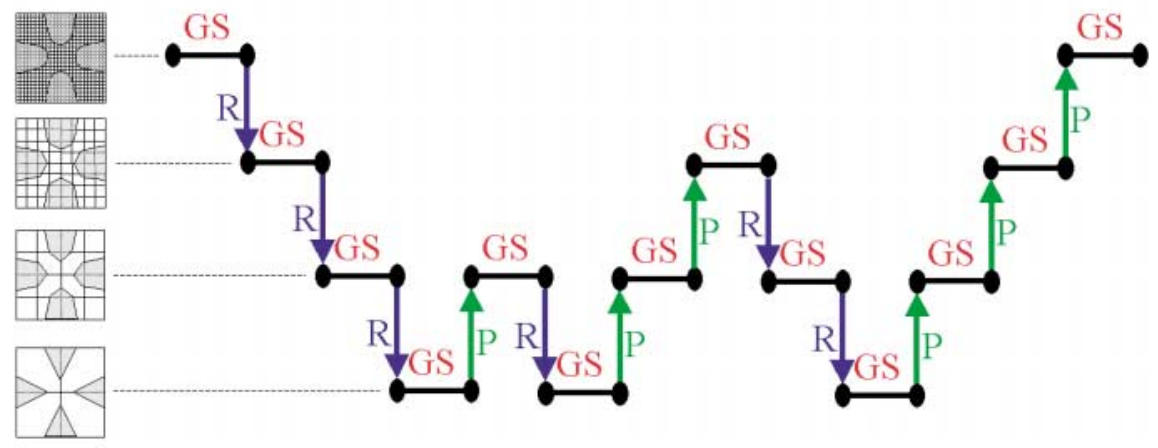

FIG. 2. (Color) Representation of the TOUTATIS cycle (GS: 3 Gauss-Seidel; R: restriction; P: prolongation). 

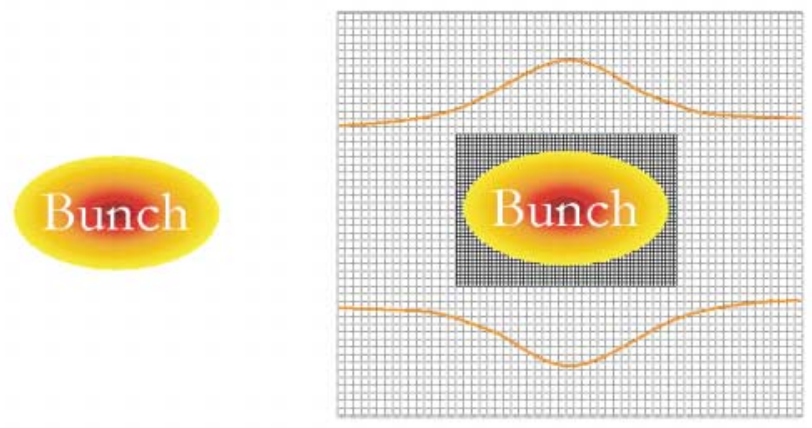

\section{Bunch}

FIG. 3. (Color) Scheme of the adaptive mesh refinement.

\section{ADAPTIVE MESH REFINEMENT}

In order to take into account neighbor bunches, the longitudinal dimension of the grid is set to $\beta \lambda^{1}$ and a longitudinal periodicity is imposed in the relaxation process. The main drawback of this technique occurs during acceleration of the bunch. As the phase spread decreases, the resolution on the bunch decreases also.

To simply solve this problem, TOUTATIS uses a second mesh which is embedded in the main grid (Fig. 3). Its dimensions are a function of bunch rms sizes while the big grid dimensions are a function of the vane geometry.

\section{TESTS}

\section{A. Theoretical comparison}

The multigrid solver has been validated with a Gaussian cylindrical beam. Figure 4 shows the radial component

\footnotetext{
${ }^{1} \beta$ is the reduced speed of the bunch, $\lambda$ is the radio frequency wavelength, and $\beta \lambda$ is the spatial periodicity of the structure.
}

of the electrical field calculated with different resolutions for the finest grid $\left(65^{3}, 33^{3}, 17^{3}, 9^{3}\right)$ compared to the theoretical value.

This test shows the good agreement achieved with this solver. The maximum discrepancy is less than $0.7 \%$ for the $65^{3}$ and $33^{3}$ cases. It is also interesting to notice that the low resolution cases give a reasonable agreement which allows very fast calculations (15 $\mathrm{min}$ ).

\section{B. Experimental comparison}

Reference [1] describes in detail an experimental confrontation between TOUTATIS and RFQ2 measurements performed in 1993 at CERN [8]. It is shown that the discrepancy is in the same region of measurement errors, around 5\%, while PARMULT discrepancy is around 15\%. PARMULT is an old version of PARMTEQM [9], the reference code for this kind of simulation. Table I shows the agreement obtained with TOUTATIS for the rms sizes.

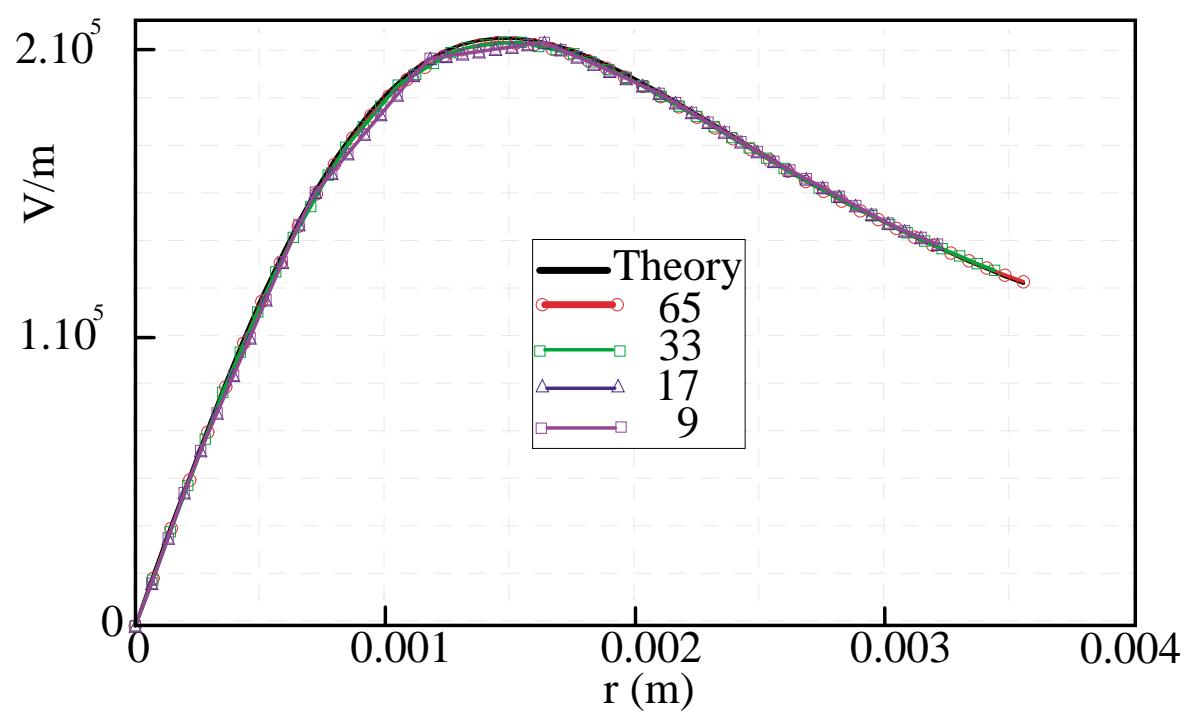

FIG. 4. (Color) Theoretical field and computed fields for different resolutions of the finest grid $\left(65^{3}, 33^{3}, 17^{3}, 9^{3}\right)$. 
TABLE I. Discrepancies, in percent, between codes and measurements for one configuration of RFQ2 tests.

\begin{tabular}{ccc}
\hline \hline rms sizes & TOUTATIS & PARMULT \\
\hline $\mathrm{X}$ & 2 & 13 \\
$\mathrm{Y}$ & 2 & 20 \\
$\mathrm{Z}$ & 5 & 9 \\
\hline \hline
\end{tabular}

\section{SIMULATION OF COUPLING GAPS}

The main advantage of the numerical approach of TOUTATIS is the possibility of simulating any vanes geometry. For example, the effect of discontinuity as the coupling gaps for segmented RFQs can be estimated. This is a very important point, especially when the geometry of these gaps (Fig. 5) is slightly complicated in order to reduce the sparking probability $[4,10]$.

To minimize the coupling gap perturbation, Young [11], from LANL, has put into practice a new technique consisting of locating the gap at the longitudinal position crossed by the bunch when the rf power is equal to zero. Applying this concept in a particular cell gives the law

$$
z=L_{c} \frac{\left|\Phi_{s}\right|}{\pi}
$$

for the position gap center, where $L_{c}$ is the cell length and $\Phi_{s}$ is the synchronous phase. A typical TOUTATIS result for the electrical potential calculation in the horizontal plane without and with a coupling gap is shown in Fig. 6.

In favor of the IPHI project, several configurations for coupling gaps have been tested especially by varying the gap width and location [12]. The significant results for the three gaps of the IPHI design are compiled in Table II. $\tilde{\epsilon}_{t}$ is the rms transverse emittance.

This study shows that the coupling gaps must be included in beam dynamics simulations to avoid forecasts that are too optimistic (emittance growth, losses, and activation) and the gap width has to be set as small as possible and the center located at Young's position.

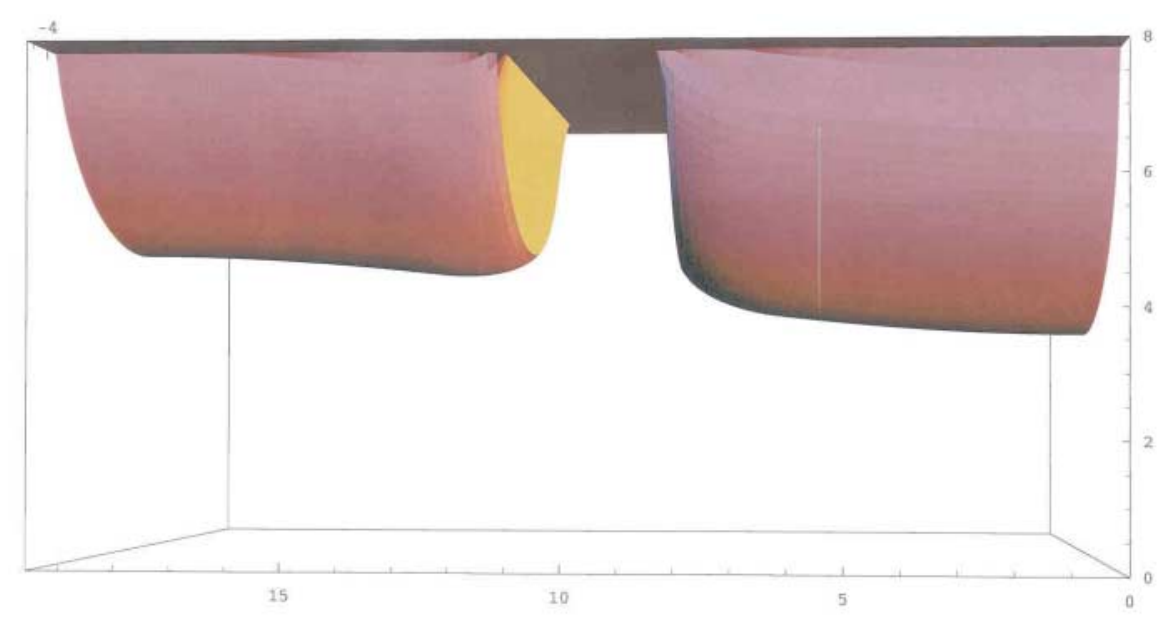

FIG. 5. (Color) Vane profile with coupling gap. An elliptical curvature avoids a field enhancement without impairing the focusing forces significantly.
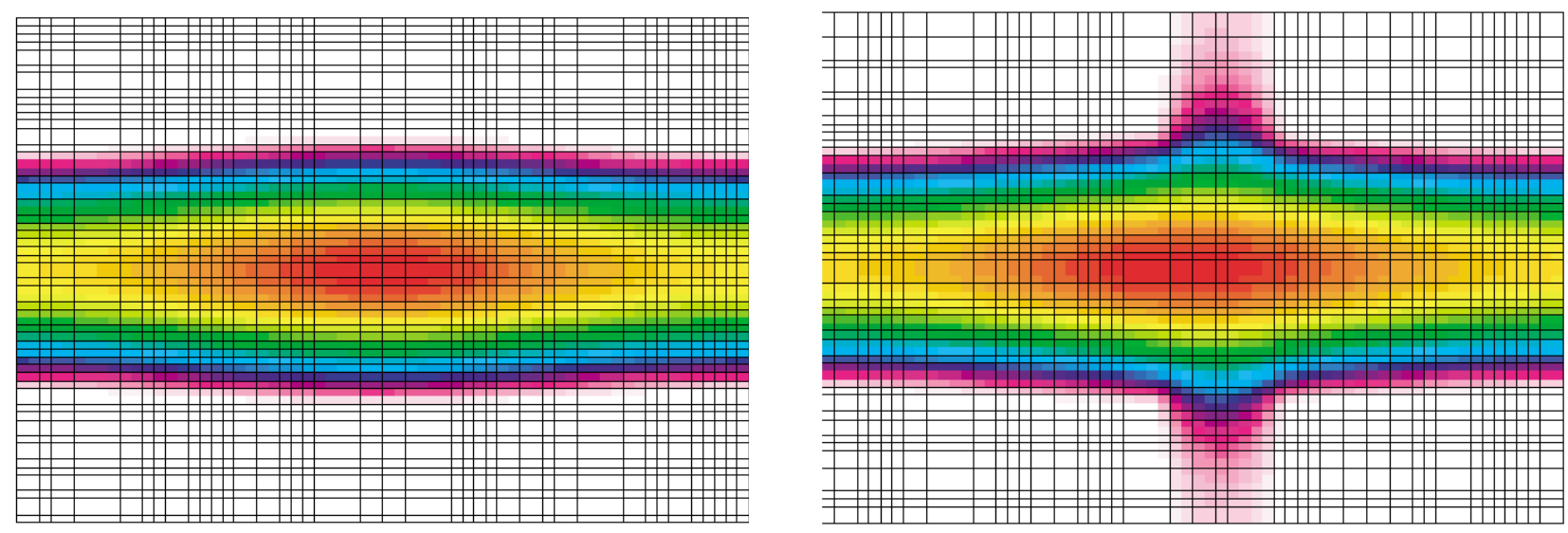

FIG. 6. (Color) Equipotentials in the horizontal plane without and with a coupling gap. 
TABLE II. Main results about gaps effects (*: gaps at exactly 2,4 , and $6 \mathrm{~m}$; $\dagger$ : gaps at Young's location).

\begin{tabular}{crrrrc}
\hline \hline Gap width $(\mathrm{mm})$ & 0 & $3.5^{*}$ & $3.5^{\dagger}$ & $2.2^{*}$ & $2.2^{\dagger}$ \\
\hline$\tilde{\boldsymbol{\epsilon}}_{t, \text { out }} / \tilde{\boldsymbol{\epsilon}}_{t, \text { in }}(\%)$ & 4 & 28 & 12 & 12 & 8 \\
Transmission $(\%)$ & 97 & 95 & 96 & 97 & 97 \\
\hline \hline
\end{tabular}

\section{CONCLUSION}

A new RFQ code for beam simulation, TOUTATIS, has been written with the goals of cross-checking the results of other codes and reaching a more reliable description of the electrical fields in the linac. Its numerical approach allows one to simulate accurately, for any vanes geometry, the whole beam zone contrary to PARMTEQM, which is limited by cylindrical harmonics $[13,14]$. The multigrid solver permits fast calculations compared to LIDOS, which uses Chebyshev acceleration [5]. An adaptive mesh refinement is implemented in order to describe as well as possible the charge distribution without impairing the computation time. TOUTATIS has also been written to be a friendly user code (multiplatforms, PARMTEQM input file can be directly used as TOUTATIS input file) and can be used by any laboratories working with CEA.

\section{ACKNOWLEDGMENTS}

I wish to acknowledge Nicolas Pichoff (CEA) and Alessandra Lombardi (CERN) for their contribution in this work.
[1] R. Duperrier, Ph.D. thesis, University of Orsay, 2000.

[2] E. Durand, Electrostatique, Méthodes de calcul, Diélectriques (Masson, Paris, 1966), Vol. III.

[3] W. Press, S. Teukolsky, W. Vetterling, and B. Flannery, Numerical Recipes, The Art of Scientific Computing (Cambridge University, Cambridge, England, 1992).

[4] R. Ferdinand, in Proceedings of the Linear Accelerator Conference, Monterey, CA, 2000 (SLAC, Stanford, CA, 2000).

[5] B. Bondarev, A. Durkin, and S. Vinogradov, in Computational Accelerator Physics, edited by J. J. Bisognano, AIP Conf. Proc. No. 391 (AIP, New York, 1997).

[6] A. Brandt, Mathematics of Computations (Cambridge University, Cambridge, England, 1977).

[7] P. Pierini, in Proceedings of the International Computational Accelerator Physics Conference, Monterey, CA, 1998 (to be published).

[8] A. Lombardi, E. Tanke, T. Wangler, and M. Weiss, CERN Technical Report No. PS93-13 (HC), 1993.

[9] K. R. Crandall, J. H. Billen, R. S. Mills, D. L. Schrage, R. H. Stokes, G. H. Neuschaefer, T. P. Wangler, and L. M. Young, LANL Technical Report No. LA-UR-96-1836, 1997.

[10] P. Balleyguier, in Proceedings of the Linear Accelerator Conference, Monterey, CA, 2000 (Ref. [4]).

[11] L. Young (private communication).

[12] R. Duperrier, CEA Technical Report No. CEA/DSM/ DAPNIA/SEA/IPHI 2000/07, 2000.

[13] K. Crandall, LANL Technical Report No. LA-9695-MS, 1983.

[14] R. Duperrier, in Proceedings of the International Linear Accelerator Conference, Chicago, 1998 (Argonne National Laboratory, Argonne, IL, 1998). 